\title{
Efficacy of a Modified Sepsis System on the Mortality Rate of Septic Shock Patients in the Emergency Department of Siriraj Hospital
}

\author{
Natvadee Rakpraisuthepsiri, B.S. ${ }^{1}$, Usapan Surabenjawong, M.D. ${ }^{2}$, Chok Limsuwat, M.D. ${ }^{2}$, \\ Ploylarp Lertvipapath, M.Sc. ${ }^{3}$
}

${ }^{1}$ Outpatient Pharmacy Division, Department of Pharmacy, Faculty of Medicine Siriraj Hospital, Bangkok Noi, Bangkok 10700, Thailand. ${ }^{2}$ Department of Emergency Medicine, Faculty of Medicine Siriraj Hospital, Mahidol University, Bangkok Noi, Bangkok 10700, Thailand. ${ }^{3}$ Adverse Drug Reaction Unit, Division of Academic Affairs, Department of Pharmacy, Faculty of Medicine Siriraj Hospital, Mahidol University, Bangkok Noi, Bangkok 10700, Thailand.

Received 31 October 2021 • Revised 21 December 2021 • Accepted 27 December 2021 • Published online 21 February 2022

\begin{abstract}
:
Objective: To compare the 28-day mortality rate between pre- and post-implementation of a modified sepsis fast-track system.

Material and Methods: A cross-sectional cohort study was conducted at the Emergency Room (ER) of Siriraj Hospital, Bangkok. All patients who were diagnosed with septic shock and who received antimicrobial treatment at admission in the ER were included.
\end{abstract}

Results: In total, 420 patients were included in the study, split into 210 patients in the pre-protocol group and 210 patients in the post-protocol group. Comparing between pre- and post-modified sepsis fast-track system implementation, the patients who received antimicrobials within 1 hour numbered $140(66.7 \%)$ and 175 (83.3\%), respectively (OR 2.5, 95\% Cl 1.57-3.97, p-value<0.001). The 28-day mortality rates of the pre- and post-protocol groups were $44.8 \%$ vs. $34.8 \%$ ( $p$-value=0.036). According to the multivariate analysis, the factors that were significantly related to $28-$ day mortality in patients with septic shock were age greater than 75 years, diabetes mellitus, and initial SOFA score $\geq 9$.

Conclusion: Implementation of a modified sepsis program improved the time to first antimicrobial administration and decreased the 28-day mortality for patients with septic shock in the ER.

Keywords: antimicrobials, sepsis program modification, septic shock

Contact: Ploylarp Lertvipapath, M.Sc.

Adverse Drug Reaction Unit, Division of Academic Affairs, Department of Pharmacy,

Faculty of Medicine Siriraj Hospital, Mahidol University, Bangkok Noi, Bangkok 10700, Thailand.

E-mail: ploylarpl@gmail.com

(c) 2022 JHSMR. Hosting by Prince of Songkla University. All rights reserved.

This is an open access article under the CC BY-NC-ND license

(http://www.jhsmr.org/index.php/jhsmr/about/editorialPolicies\#openAccessPolicy).
J Health Sci Med Res 2022;40(5):543-550 doi: $10.31584 /$ jhsmr.2022865 www.jhsmr.org 


\section{Introduction}

Sepsis is a potentially life-threatening condition that causes multi-organ dysfunction through the dysregulated host response to infection. Organ dysfunction can be defined by the Sequential Organ Failure Assessment (SOFA) score, in which a higher score is associated with a greater inhospital mortality rate. ${ }^{1}$ Sepsis remains one of the major global health burdens in terms of morbidity, mortality, and resource utilization. In 2017, an estimated 48.9 million incident of sepsis were recorded worldwide and 11.0 million sepsis-related deaths were reported, representing $19.7 \%$ of all global deaths. ${ }^{2}$ In Thailand, the mortality rate of sepsis and septic shock are $34.3 \%$ and $52.6 \%$ that higher than the global data. ${ }^{3}$

Early resuscitation and hemodynamic support, especially the early initiation of appropriate antimicrobials, are important strategies to improve the outcome of sepsis patients. ${ }^{4}$ The administration of antimicrobials should be done as soon as possible after diagnosis. ${ }^{1}$ Each hour of delayed administration of antimicrobials increases the risk of death. One study reported that the mortality rate among patients who received antimicrobials within 1 hour postdiagnosis was statistically significantly lower than in patients who had antimicrobial therapy initiated 6 hour or more post-diagnosis $(24.6 \%$ and $33.1 \%$, respectively, p-value< 0.001). ${ }^{5}$

The Third International Consensus Definitions for Sepsis and Septic Shock (Sepsis-3) defines sepsis as lifethreatening organ dysfunction caused by a dysregulated host response to infection. ${ }^{1}$ The Surviving Sepsis Campaign's 2021 international guidelines for management of sepsis and septic shock recommend that appropriate antimicrobial therapy should be administered immediately, ideally within 1 hour post-diagnosis to septic shock or possible septic shock patients. ${ }^{6,7}$

Despite strong evidence suggesting that the early initiation of appropriate antimicrobial treatment within 1 hour is associated with a lower mortality rate, in our setting, data on the initiation of appropriate antibiotic treatment within 1 hour post-diagnosis are quite limited. Indeed, the time lapsed from diagnosis to initiation of appropriate antimicrobial treatment can often be several hours due to the complicated drug-ordering system in our institution. When a patient was diagnosed with septic shock, the doctor prescribed an antimicrobial and sent the prescription to a pharmacist. Then, the pharmacist would complete the prescription, take payment, and dispense the medicine before the drug could be administered to the patient. For the purpose of reducing the drug administration time, the researchers implemented a new fast-track sepsis system with an aim to shorten the process and time, as shown in Figure 1. To ensure the quality of this system, the researchers conducted a study at Siriraj Hospital where this new system was tried in order to assess the reduction, if any, in 28-day mortality after the system implementation.

\section{Material and Methods}

The study design was a cross-sectional cohort study which aimed to measure the impact of a modified sepsis protocol on the mortality rate in an ER from septic shock and sepsis. The study was conducted at ER of Siriraj Hospital in Bangkok, Thailand. The modified protocol (Figure 1) was implemented in December 2018. Participants were enrolled from the ER. Relevant data were obtained from the medical records and computerized system of the hospital for patients diagnosed with septic shock. The inclusion criteria were patients aged at least 18 years who were diagnosed with septic shock and who received antimicrobials in the ER. The exclusion criteria were patients who received antimicrobials before entering the ER. This study was approved by the Siriraj Institutional Review Board. After treatment and discharge from the ER, our study collected hospital outcomes including mortality in hospital and 28-day mortality. 
Treatment flow for patients with diagnosis of septic shock in the 2 study groups

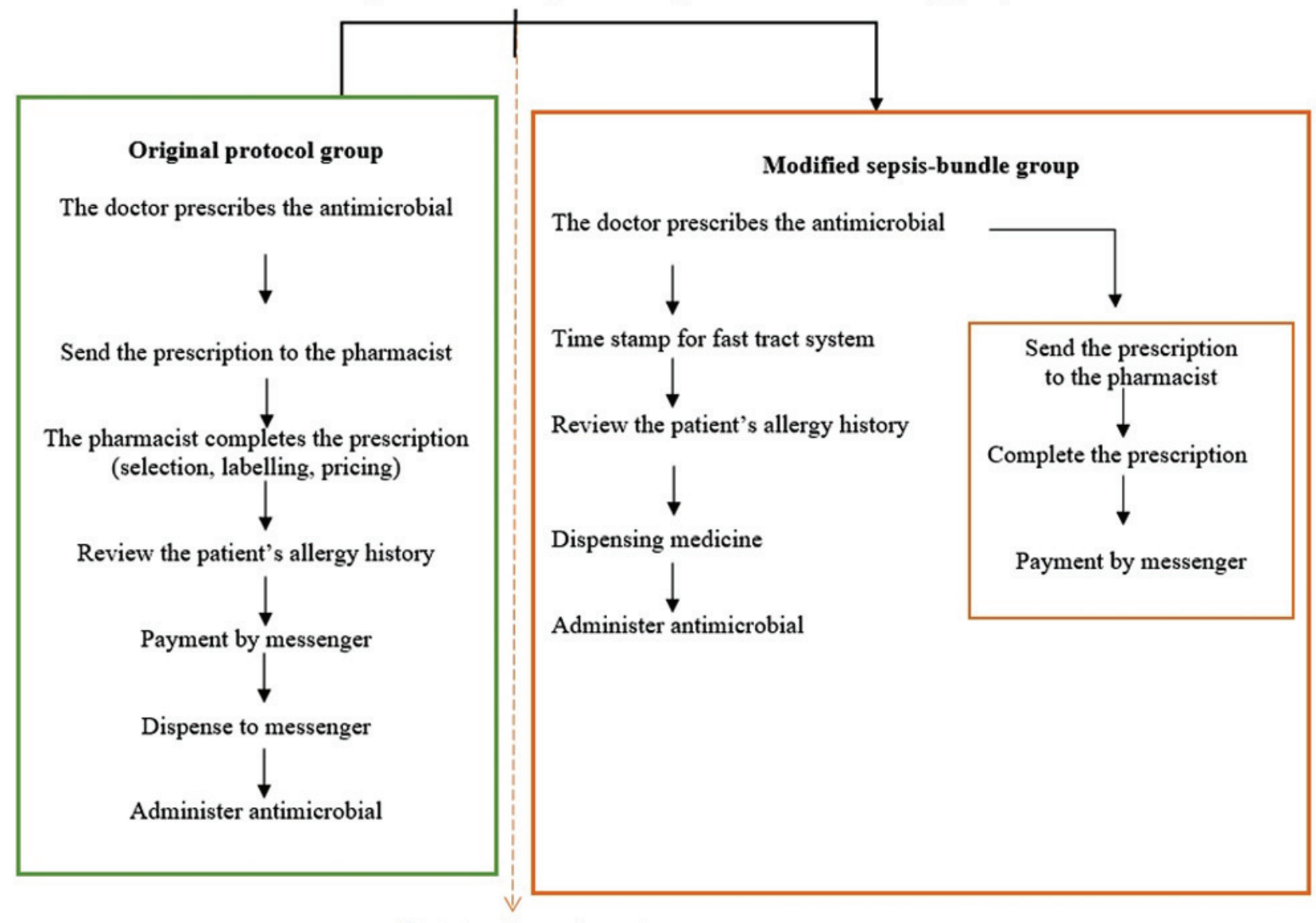

Fast-track sepsis system

Figure 1 Fast-track sepsis system modification compare with previous sepsis

The modified sepsis protocol is a new dispensing system which is aimed to reduce the antimicrobials administration time. To shorten the process, the new protocol cut off and payment process by using the parallel track. After the fast track is stamped, the attending staff would carefully order for empirical antimicrobials and send the prescription to the pharmacist. The allergy reviewing and antimicrobial prescribing would be done immediately. At the same time, the parallel payment process would be done by the relatives of the patient. 
Septic shock was defined as persistent hypotension requiring a vasopressor to maintain mean arterial pressure $\geq 65 \mathrm{mmHg}$ despite adequate volume resuscitation.

Appropriate empirical antimicrobial therapy was defined based on the following parameters:

1. Empirical antimicrobial therapy was administered within the time limit of 1 hour.

2. First empirical antimicrobial dosage was delivered as a full dose.

3. The dose and frequency of the empirical antimicrobial administration were adjusted in patients with renal insufficiency.

4. De-escalating antimicrobial was administered at a certain dose, and then possible the dose was lowered. Mortality rate referred to all-cause mortality. We collected this data from the in-patient and follow up documentation.

The primary outcome was the 28-day mortality rate compared between the two study periods (pre- and postprotocol implementation). The secondary outcomes were the appropriateness of antimicrobial use, the in-hospital mortality rate, the rate of systemic inflammatory response syndrome (SIRS) resolution, and factors associated with the 28-day mortality.

The sample size was calculated based on the mortality rate, as reported in 2018 in Siriraj Hospital. The researchers predicted that the sepsis program modification would reduce the mortality rate by $10.0 \%$. After calculation, the sample size of 420 patients was considered sufficient for achieving $80.0 \%$ power, with a significance level (alpha) of 0.05 .

Descriptive data, which are the categorical data, are reported by number and percentage. The continuous data are presented as median and interquartile range (IQRs). Chi-square and Mann-Whitney $U$ test were used to compare differences between the groups. SPSS ${ }^{\circledR}$ version 22.0 for Windows ${ }^{\circledR}$ was used for the statistical analysis, descriptive analysis, and inferential analysis. The statistical significance level was defined as $p-$ value $<0.050$.

\section{Results}

In total, 420 patients were included in this study, comprising 210 patients in the pre-protocol period group and 210 in the post-protocol period. The baseline characteristics of the overall patients are shown in Table 1. The median age was $73 \pm 21$ years and $51.4 \%$ were male. Most of the patients were from a community setting. When comparing between the 2 groups, although the patients who were in the post-protocol group, i.e., post-implementation of the fast-track sepsis system, had a lower median SOFA score, they had higher numbers of patients with renal disease, respiratory and cardiovascular failure (Table 1). The most common source of infection in both groups was pneumonia. The post-implementation group had higher rates of urinary tract and intra-abdominal infections. In addition, we found no differences in specimen blood culture data between the previous and modified systems (35.9\% vs $39.7 \%, p-v a l u e=0.463)$. The utilization of a vasopressor for shock resuscitation was not different between the 2 groups (139 vs 134 cases in the pre- and post-protocol groups, respectively). Moreover, we found no differences in vasopressor usage between the previous and modified systems. When specific name of vasopressor, norepinephrine and epinephrine showed different between pre-protocol and post-protocol (66.5\% vs 63.5\%, p-value $=0.562$ ). In the subgroup of intra-abdominal infection, we found no difference in the numbers of patients requiring surgery between the previous and modified systems (13.4\% vs $23.1 \%, p$-value $=0.181$ ).

For the primary outcome, the 28-day mortality rate was $34.8 \%$ in the post-protocol group against $44.8 \%$ in the pre-protocol group, which was significantly lower ( $p$-value= 0.036). The in-hospital death in the post-protocol group was also lower than in the pre-protocol group $(p-v a l u e=$ 0.011), although the SIRS resolution was not statistically different between the 2 groups (Table 2).

Regarding the appropriateness of the antimicrobial administration in the study, the researchers found that 
success in achieving antibiotics administration within $1 \mathrm{~h}$ was $83.3 \%$ in the post-protocol group, which was significantly higher (OR 2.5, 95\% Cl 1.57-3.97, p-value $<0.001$ ) than in pre-protocol group. We found that the loading antimicrobial dose administered in the post-protocol group was higher than in the pre-protocol group (OR 3.05, 95\% Cl 1.26-7.38, $p$-value $=0.010)$. The adjusted renal dose was also higher number of patients in the post-protocol group (70.5 vs.
$82.4 \%, p-v a l u e=0.004)$. Only the results from de-escalation of the antimicrobial administration were higher in the preprotocol sepsis group (Table 3).

The univariate analysis factors associated with 28day mortality were age $>75$ years, liver disease, diabetes mellitus, SOFA score $\geq 9$, Fast-track Sepsis Program administration, adjusted renal dose, and de-escalation of antimicrobial administration (Table 4).

Table 1 Baseline characteristics of the study patients

\begin{tabular}{|c|c|c|c|c|}
\hline Characteristic & $\begin{array}{l}\text { Total } \\
\text { No. }(\%) \\
(n=420)\end{array}$ & $\begin{array}{l}\text { Previous system } \\
\text { No. }(\%) \\
(n=210)\end{array}$ & $\begin{array}{l}\text { Modified system } \\
\text { No. }(\%) \\
(n=210)\end{array}$ & p-value \\
\hline Male & $216(51.4)$ & $117(55.7)$ & $99(47.1)$ & 0.079 \\
\hline Age (median $\pm \mid Q R)$ & $73 \pm 21$ & $73 \pm 24$ & $73 \pm 20$ & \\
\hline$<65$ & $145(34.5)$ & $85(40.5)$ & $60(28.6)$ & \\
\hline $65-80$ & $171(40.7)$ & $85(40.5)$ & $86(41.0)$ & 0.007 \\
\hline$>80$ & $104(24.8)$ & $40(19.0)$ & $64(30.5)$ & \\
\hline \multicolumn{5}{|l|}{ Habitation } \\
\hline Home & $385(91.7)$ & 205 (97.6) & $183(87.1)$ & $<0.001$ \\
\hline Nursing care & $35(8.3)$ & $5(2.4)$ & $27(12.9)$ & \\
\hline \multicolumn{5}{|l|}{ Underlying disease } \\
\hline DM & $148(35.2)$ & $71(33.8)$ & $77(36.7)$ & 0.540 \\
\hline Cardiovascular disease & $115(27.4)$ & $51(24.3)$ & $64(30.5)$ & 0.155 \\
\hline Renal disease & $108(25.7)$ & $42(20.0)$ & $66(31.4)$ & 0.007 \\
\hline Liver disease & $68(16.2)$ & $35(16.7)$ & $33(15.7)$ & 0.791 \\
\hline \multicolumn{5}{|l|}{ Immunosuppressive drug } \\
\hline Corticosteroids & $14(3.3)$ & $4(1.9)$ & $10(4.8)$ & 0.103 \\
\hline Immunoglobulin suppressant & $5(1.2)$ & $0(0.0)$ & $5(2.4)$ & 0.204 \\
\hline Vasopressor & $273(65.2)$ & $139(66.5)$ & $134(63.5)$ & 0.562 \\
\hline Norepinephrine & $261(95.6)$ & $128(49.0)$ & $133(50.1)$ & \\
\hline Dopamine & $3(1.1)$ & $2(1.4)$ & $1(0.7)$ & \\
\hline Epinephrine & $9(3.3)$ & $9(6.5)$ & 0 & \\
\hline \multicolumn{5}{|l|}{ Source of infection } \\
\hline Respiratory tract & $141(33.6)$ & $66(31.4)$ & $75(35.7)$ & 0.352 \\
\hline Urinary tract & $106(25.2)$ & $40(19.0)$ & $66(31.4)$ & 0.003 \\
\hline Intra-abdominal & $121(28.8)$ & $82(39.0)$ & $39(18.6)$ & $<0.001$ \\
\hline Specimen blood culture & $135(38.1)$ & $52(35.9)$ & $83(39.7)$ & 0.463 \\
\hline qSOFA score $\geq 2$ & $309(73.6)$ & $166(79.0)$ & $143(68.1)$ & 0.011 \\
\hline SOFA score (median+IQR) & $5 \pm 2$ & $6 \pm 2$ & $3.5 \pm 2$ & \\
\hline SOFA $\geq 9$ & $54(12.9)$ & $42(20.0)$ & $12(5.7)$ & $<0.001$ \\
\hline \multicolumn{5}{|l|}{ Organ failure } \\
\hline Renal & $46(11.0)$ & $19(9.0)$ & 27 (12.9) & 0.271 \\
\hline Respiratory & $18(4.3)$ & $3(1.4)$ & $15(7.1)$ & 0.004 \\
\hline Cardiovascular & $16(3.8)$ & $3(1.4)$ & $13(6.2)$ & 0.011 \\
\hline Liver & $15(3.6)$ & $6(2.9)$ & $9(4.3)$ & 0.430 \\
\hline
\end{tabular}

$\mathrm{DM}=$ diabetes mellitus, qSOFA=quick Sequential Organ Failure Assessment, SOFA=Sequential Organ Failure Assessment, IQR=interquartile range 
Table 2 Association between the fast track sepsis system and the clinical outcomes

\begin{tabular}{|c|c|c|c|c|c|}
\hline Clinical outcome & $\begin{array}{l}\text { Total } \\
\text { No. }(\%) \\
(n=420)\end{array}$ & $\begin{array}{l}\text { Previous system } \\
\text { No. }(\%) \\
(n=210)\end{array}$ & $\begin{array}{l}\text { Modified system } \\
\text { No. }(\%) \\
(\mathrm{n}=210)\end{array}$ & $\begin{array}{l}\text { OR } \\
(95 \% \mathrm{CI})\end{array}$ & $p$-value \\
\hline 28-day mortality & $167(39.8)$ & $94(44.8)$ & $73(34.8)$ & $\begin{array}{l}0.658 \\
(0.444-0.974)\end{array}$ & 0.036 \\
\hline In-hospital death & $147(35.0)$ & $86(41.0)$ & $61(29.0)$ & $\begin{array}{l}0.59 \\
(0.394-0.888)\end{array}$ & 0.011 \\
\hline \multicolumn{6}{|l|}{ Resolution of SIRS } \\
\hline No & $97(23.1)$ & $53(25.2)$ & $44(21.0)$ & 1.27 & 0.297 \\
\hline Yes & $323(76.9)$ & $157(74.8)$ & $166(79.0)$ & $(0.808-2.008)$ & \\
\hline
\end{tabular}

SIRS=systemic inflammatory response syndrome

Table 3 Appropriateness of the antimicrobial therapy in the study

\begin{tabular}{|c|c|c|c|c|c|}
\hline Antimicrobial criteria & $\begin{array}{l}\text { Total } \\
\text { No. }(\%) \\
(n=420)\end{array}$ & $\begin{array}{l}\text { Previous system } \\
\text { No. }(\%) \\
(n=210)\end{array}$ & $\begin{array}{l}\text { Modified system } \\
\text { No. }(\%) \\
(n=210)\end{array}$ & $\begin{array}{l}\text { OR } \\
(95 \% \mathrm{Cl})\end{array}$ & p-value \\
\hline Antimicrobial within 1 hour & $315(75.0)$ & $140(66.7)$ & 175 (83.3) & $\begin{array}{l}2.5 \\
(1.57-3.97)\end{array}$ & $<0.001$ \\
\hline Loading dose administration & $393(93.6)$ & $190(90.5)$ & $203(96.7)$ & $\begin{array}{l}3.053 \\
(1.26-7.38)\end{array}$ & 0.010 \\
\hline \multicolumn{6}{|l|}{ Adjusted renal dose } \\
\hline $\begin{array}{l}\text { No } \\
\text { Yes }\end{array}$ & $\begin{array}{l}99(23.6) \\
321(76.4)\end{array}$ & $\begin{array}{l}62(29.5) \\
148(70.5)\end{array}$ & $\begin{array}{l}37(17.6) \\
173(82.4)\end{array}$ & $\begin{array}{l}1.959 \\
(1.23-3.11)\end{array}$ & 0.004 \\
\hline $\begin{array}{l}\text { De-escalation of antimicrobial } \\
\text { use }\end{array}$ & $280(79.1)$ & $157(96.3)$ & $123(64.4)$ & $\begin{array}{l}0.69 \\
(0.03-0.17)\end{array}$ & 0.001 \\
\hline
\end{tabular}

Table 4 Univariate analysis of the factors associated with 28-day mortality

\begin{tabular}{|c|c|c|c|c|}
\hline \multirow{2}{*}{ Factor } & \multirow{2}{*}{$\begin{array}{l}\text { Total }(\%) \\
(n=420)\end{array}$} & \multirow{2}{*}{$\begin{array}{l}\text { Death (\%) } \\
(n=167)\end{array}$} & \multicolumn{2}{|c|}{ Univariate logistic regression } \\
\hline & & & OR $(95 \% \mathrm{Cl})$ & p-value \\
\hline Age $>75$ years & 186 & $89(47.8)$ & $\begin{array}{l}1.84 \\
(1.24-2.73)\end{array}$ & 0.003 \\
\hline Liver disease & 68 & $40(58.8)$ & $\begin{array}{l}2.53 \\
(1.49-4.29)\end{array}$ & 0.001 \\
\hline Diabetes & 148 & $73(49.3)$ & $\begin{array}{l}1.84 \\
(1.23-2.77)\end{array}$ & 0.003 \\
\hline SOFA score $\geq 9$ & 54 & $33(61.1)$ & $\begin{array}{l}2.72 \\
(1.51-4.89)\end{array}$ & 0.001 \\
\hline \multicolumn{5}{|l|}{ Fast-track sepsis program } \\
\hline Pre & 210 & $94(44.8)$ & 0.66 & 0.037 \\
\hline Post & 210 & $73(34.8)$ & $(0.44-0.97)$ & \\
\hline Adjusted renal dose & 198 & $80(40.4)$ & $\begin{array}{l}0.53 \\
(0.34-0.84)\end{array}$ & 0.007 \\
\hline De-escalation of antimicrobial use & 280 & $81(28.9)$ & $\begin{array}{l}0.45 \\
(0.27-0.77)\end{array}$ & 0.003 \\
\hline
\end{tabular}

SOFA=Sequential Organ Failure Assessment 
Table 5 Multivariate analysis of factors associated with clinical outcomes on 28-day mortality

\begin{tabular}{|c|c|c|c|c|c|}
\hline Factor & $\begin{array}{l}\text { Total }(\%) \\
(n=420)\end{array}$ & $\begin{array}{l}\text { Survived (\%) } \\
(n=253)\end{array}$ & $\begin{array}{l}\text { Deceased (\%) } \\
(n=167)\end{array}$ & OR $(95 \% \mathrm{Cl})$ & $\mathrm{p}$-value \\
\hline Age $>75$ years & 186 & 97 (52.2) & $89(47.8)$ & $\begin{array}{l}2.08 \\
(1.36-3.19)\end{array}$ & 0.001 \\
\hline Diabetes & 148 & 75 (50.7) & 73 (49.3) & $\begin{array}{l}2.02 \\
(1.32-3.11)\end{array}$ & 0.001 \\
\hline SOFA score $\geq 9$ & 54 & 21 (38.9) & $33(61.1)$ & $\begin{array}{l}3.73 \\
(1.94-7.19)\end{array}$ & $<0.001$ \\
\hline
\end{tabular}

SOFA=Sequential Organ Failure Assessment

Following multivariate analysis, the factors that were related to the 28 -day mortality were age $>75$ years, diabetes mellitus, and SOFA score $\geq 9$ (Table 5).

\section{Discussion}

The modified sepsis system that the researchers helped introduce in Siriraj Hospital to attempt to increase the rate of within 1 hour antimicrobial administration decreased the 28-day and in-hospital mortality rates significantly. The results are compatible with previous studies which found that appropriate antimicrobial therapy were associated with a survival rate of $50.0-80.0 \% .^{7,8}$ Our data strongly support the findings of these studies, that the initiation of antimicrobial administration within 1 hour was associated with a substantially decreased mortality rate in patients with septic shock.

Other than the Surviving Sepsis Campaign international consensus guideline recommendation of beginning effective antimicrobial therapy within the first hour ${ }^{9}$, a previous study also found that each hour of delayed antimicrobial therapy after a patient developed hypotension was associated with an average decrease in survival of $7.6 \%{ }^{7}$ In addition, Gaieski et al. found that antimicrobial administration within 1 hour showed a statistically significant associated decrease in the mortality rate of $13.7 \%$ and the time from qualification for early goal-directed therapy to antimicrobial administration within 1 hour showed a statistically significant associated decrease in mortality rate of $13.5 \%$. $^{10}$

The intervention of the fast-track sepsis program is a new method in our institution, which aims to improve the pharmacist service to make it more lean and able to hasten the speed of delivery of antimicrobial administration. This modification has lead to better treatment and fewer delays. After the intervention, the achievement rate of meeting the target time of within 1 hour antimicrobial administration was $83.3 \%$, which was higher than with the previous system. Besides improved clinical outcomes, the 28-day mortality was also significantly decreased. This could imply that the availability of this fast-track sepsis system led to better clinical outcomes in patients with septic shock.

According to the multivariate analysis, the factors pertaining to a higher mortality rate were age greater than 75 years, diabetes mellitus, and SOFA score greater than 9. These findings are similar to previous studies, which reported that higher age, more underlying diseases, and higher SOFA scores were related to poor outcomes ${ }^{1}$. However, the modified fast-track system is not the only direct factor that leads to better survival, as a patient may receive the antimicrobial therapy faster, there are many factors that are important for survival, for example, the severity of their disease. 
The strength of this study is its prospective design. There were no missing data in the outcomes that we were interested in studying. The other benefit is its generalizability. Although the data were from a single center, the study was conducted in the Emergency Department, which has a population of both medical and surgical patients. These are the same types of patients which can be found in other hospitals in Thailand. Thus, they could apply this system to their hospitals too.

A limitation of this study to note is the absence of resuscitation data, which is be an important factor relating to the survival of septic shock patients. However, Siriraj Hospital is a high-quality university hospital and we believe that the standard of treatment was not different between our 2 groups. Another limitation of this study is that the postprotocol group had lower SOFA scores when compared with the pre-protocol group, which could have impacted the mortality of the primary outcome. Further investigations with larger populations are needed to identify the power of the new protocol.

\section{Conclusion}

This study illustrates the potential advantages of implementing a fast-track sepsis program modification. A fast-track sepsis had the effect of decreasing the initiation time of antimicrobial therapy, resulting in decreased inhospital and 28-day mortality rates of septic shock patients in our study. However, further studies are needed to prove these clinical benefits.

\section{Acknowledgement}

The authors are grateful to the patients of the Emergency Department, Siriraj Hospital, who participated in this study.

\section{Conflict of interest}

There are no potential conflicts of interest to declare.

\section{References}

1. Singer M, Deutschman CS, Seymour CW, Shankar-Hari M, Annane D, Bauer $M$, et al. The third international consensus definitions for sepsis and septic shock (Sepsis-3). JAMA 2016; 315:801-10.

2. Rudd KE, Johnson SC AK, Shackelford KA, Tsoi D, Kievlan DR, et al. Global, regional, and national sepsis incidence and mortality, 1990-2017: analysis for the global burden of disease study. Lancet 2020;395:200-11.

3. Angkasekwinai $\mathrm{N}$, Rattanaumpawan $\mathrm{P}$, Thamlikitkul V. Epidemiology of sepsis in Siriraj Hospital 2007. J Med Assoc Thai 2009;92:S68-78.

4. Rivers E, Nguyen B, Havstad S, Ressler J, Muzzin A, Knoblich $B$, et al. Early goal-directed therapy collaborative group. Early goal-directed therapy in the treatment of severe sepsis and septic shock. N Engl J Med 2001;345:1368-77.

5. Ferrer R, Martin-Loeches I, Phillips G, Osborn TM, Townsend S, Dellinger RP, et al. Empiric antibiotic treatment reduces mortality in severe sepsis and septic shock from the first hour: results from a guideline-based performance improvement program. Crit Care Med 2014;42:1749-55.

6. Evans L, Rhodes A, Alhazzani W, Antonelli M, Coopersmith CM, French $C$, et al. Surviving sepsis campaign: international guidelines for management of sepsis and septic shock 2021. Intensive Care Med 2021;47:1181-247.

7. Kumar A, Roberts D, Wood KE, Light B, Parrillo JE, Sharma $S$, et al. Duration of hypotension before initiation of effective antimicrobial therapy is the critical determinant of survival in human septic shock. Crit Care Med 2006;34:1589-96.

8. Kumar A, Ellis P, Arabi Y, Roberts D, Light B, Parrillo JE, et al. Initiation of inappropriate antimicrobial therapy results in a fivefold reduction of survival in human septic shock. Chest 2009;136:1237-48.

9. Levy MM, Evans LE, Rhodes A. The surviving sepsis campaign bundle: 2018 update. Crit Care Med 2018;46:997-1000.

10. Gaieski DF, Pines JM, Band RA, Mikkelsen ME, Massone R, Furia FF, et al. Impact of time to antibiotics on survival in patients with severe sepsis or septic shock in whom early goaldirected therapy was initiated in the emergency department. Crit Care Med 2010;38:1-9. 